\title{
-NOTES-
}

\section{FREQUENCIES OF LONGITUNDINAL OSCILLATION OF A NON-UNIFORM BAR*}

\author{
By G. J. HABETLER, G. H. HANDELMAN, J. R. McLAUGHLIN,
}

E. H. ROGERS, AND L. RUBENFELD

(Rensselaer Polytechnic Institute)

1. Introduction. An informal discussion of a paper by Tsiu [1] on longitudinal wave propagation in a bar of finite length but varying cross-section has led us to consider somewhat more elementary questions arising in the study of the longitudinal oscillations of such a system. If the time-harmonic displacement in the $x$-direction is denoted by $u(x) \exp (i \omega t)$, the governing differential equation is

$$
\left(A E u_{x}\right)_{x}=-m A \omega^{2} u,
$$

where $A=A(x)$ is the variable cross-sectional area, $E$ is Young's modulus, assumed to be constant, and $m$ is the mass per unit volume. We shall assume throughout that $A(x)>0$, and for the present, at least, $m$ is taken to be constant. If the ends, $x=0$ and $x=l$, are considered to be fixed, we have the boundary conditions $u(0)=u(l)=0$, whereas, if the ends are free, $u_{x}(0)=u_{x}(l)=0$. We ask, for each of these two sets of boundary conditions, whether we can characterize the changes in the natural frequencies $\omega$ which would be produced by changes in the cross-section $A(x)$.

It is more convenient to introduce the nondimensional variables $\xi=x / l, v(\xi)=u(x) / l$, $a(\xi)=A(x) / l^{2}$, and $\lambda=\left(m l^{2} \omega^{2}\right) / E$. We find that the differential equation becomes

$$
\left(a v^{\prime}\right)^{\prime}+\lambda a v=0, \quad 0<\xi<1,
$$

where denotes differentiation with respect to $\xi$. For fixed ends, the boundary conditions are

$$
v(0)=v(1)=0
$$

and for free ends, they are

$$
v^{\prime}(0)=v^{\prime}(1)=0 .
$$

The usual approach for studying the effect of changes in $a$ on the eigenvalues $\lambda$ would be the consideration of the corresponding Rayleigh quotient, $R[v]$, and the subsequent application of the usual comparison relations. We find that the Rayleigh quotient for either the eigenvalue problem given by (1) and (2) or that described by (1) and (2') is

$$
R[v]=\int_{0}^{1} a v^{\prime 2} d \xi / \int_{0}^{1} a v^{2} d \xi .
$$

- Received January 10, 1970 
We see, unfortunately, that $a(\xi)$ appears in both the numerator and denominator of Eq. (3) and the normal comparison techniques will not be useful.

2. The transformed problem. The ambiguity just noted can be removed in both the free-end and fixed-end cases by applying appropriate transformations. Consider first the fixed-end case. By applying one of the standard transformations [2, p. 292] used in Sturm-Liouville problems, namely, setting $v=w / a^{1 / 2}$, it can be shown by elementary calculations that Eq. (1) becomes

$$
w^{\prime \prime}+\left[\lambda-\left(a^{1 / 2}\right)^{\prime \prime} /\left(a^{1 / 2}\right)\right] w=0 .
$$

The boundary conditions (2) are unchanged, namely,

$$
w(0)=w(1)=0 .
$$

Further, multiplying Eq. (4) by $w$, integrating from 0 to 1, and applying integrations by parts, we find that

$$
\lambda=\int_{0}^{1}\left[{w^{\prime 2}}^{2}+\left(\left\{a^{1 / 2}\right\}^{\prime \prime} / a^{1 / 2}\right) w^{2}\right] d \xi / \int_{0}^{1} w^{2} d \xi=R[w] .
$$

In addition, if we take any two functions $v$ and $w$ which vanish at $\xi=0$ and $\xi=1$ and are related by $v=w / a^{1 / 2}$, we find that

$$
\int_{0}^{1} a{v^{\prime}}^{2} d \xi=\int_{0}^{1}\left[w^{\prime 2}+\left(\left\{a^{1 / 2}\right\}^{\prime \prime} / a^{1 / 2}\right)\right] w^{2} d \xi .
$$

Thus, the numerator and denominator in the Rayleigh quotient $R[w]$ are positive definite and we may apply the usual comparison theorems [2, p. 407]. It is assumed, of course, that the solutions of the corresponding minimum problems exist.

Specifically, consider a second eigenvalue problem given by

$$
\begin{gathered}
w^{\prime \prime}+\left[\mu-\left(b^{1 / 2}\right)^{\prime \prime} /\left(b^{1 / 2}\right)\right] w=0, \\
w(0)=w(1)=0 .
\end{gathered}
$$

Let us put the eigenvalues of the original problem in increasing order, $\lambda_{1}, \lambda_{2}, \cdots$, and similarly $\mu_{1}, \mu_{2}, \cdots$ for the problem defined by Eqs. (7) and (8). We thus find that if

$$
\left(a^{1 / 2}\right)^{\prime \prime} /\left(a^{1 / 2}\right) \leqq\left(b^{1 / 2}\right)^{\prime \prime} /\left(b^{1 / 2}\right) \text { for } 0<\xi<1 \text {, }
$$

then

$$
\lambda_{n} \leqq \mu_{n}, \text { for } n=1,2, \cdots,
$$

since the corresponding Rayleigh quotients satisfy the same inequality.

If the same transformation were to be applied to the free-end case, that is, the boundary value problem described by Eqs. (1) and (2'), it would be found that the corresponding Rayleigh quotient would no longer be susceptible to such simple interpretations. Making an analogous transformation, however, we set $w=v^{\prime} a^{1 / 2}$. We find, by elementary calculations, that Eq. (1) becomes

$$
w^{\prime \prime}+\left[\lambda^{*}-\left(a^{-1 / 2}\right)^{\prime \prime} /\left(a^{-1 / 2}\right)\right] w=0,
$$

where ${ }^{*}$ denotes the eigenvalues for the free-end case. Furthermore, the boundary condition $\left(2^{\prime}\right)$ becomes

$$
w(0)=w(1)=0 .
$$


Reasoning analogous to that above allows us to compare the eigenvalues of this last equation with the eigenvalues of a second problem

$$
w^{\prime \prime}+\left[\mu^{*}-\left(b^{-1 / 2}\right)^{\prime \prime} /\left(b^{-1 / 2}\right)\right] w=0, \quad w(0)=w(1)=0 ;
$$

that is, if the eigenvalues of the two problems in increasing order are $\lambda_{1}^{*}, \lambda_{2}^{*}, \cdots$ and $\mu_{1}^{*}, \mu_{2}^{*}, \cdots$, and if

$$
\left(a^{-1 / 2}\right)^{\prime \prime} /\left(a^{-1 / 2}\right) \leqq\left(b^{-1 / 2}\right)^{\prime \prime} /\left(b^{-1 / 2}\right) \text { for } 0<\xi<1,
$$

then $\lambda_{n}^{*} \leqq \mu_{n}^{*}$, for $n=1,2, \cdots$.

In addition to comparing eigenvalues for two fixed-end problems or two free-end problems, it might be of interest to compare the eigenvalues arising from the free-end and fixed-end problems associated with the same cross-section shape. In order to make such a comparison, we first note that

$$
\left(a^{1 / 2}\right)^{\prime \prime} /\left(a^{1 / 2}\right)-\left(a^{-1 / 2}\right)^{\prime \prime} /\left(a^{-1 / 2}\right)=(\log a)^{\prime \prime} .
$$

Comparing the Rayleigh quotients for each problem, it is easily seen that if $(\log a)^{\prime \prime} \geqq 0$, then $\lambda_{n}^{*} \leqq \lambda_{n}$, and if $(\log a)^{\prime \prime} \leqq 0$, then $\lambda_{n} \leqq \lambda_{n}^{*}$.

3. Examples and comments. Let us first observe that the results noted above for the fixed-end problem can be extended to the case of variable density. In such circumstances, Eq. (1) is replaced by $\left(a v^{\prime}\right)^{\prime}+\lambda a \rho(\xi) v=0$. For the fixed-end problem, the numerator in the Rayleigh quotient remains the same but the denominator becomes $\int_{0}^{1} a \rho w^{2} d \xi$. The conclusions previously found are therefore unchanged.

For the free-end problem, if one makes the transformation $w=(a / \rho)^{1 / 2} v^{\prime}$, Eq. (1) becomes

$$
w^{\prime \prime}+\left\{\lambda \rho-\left[(a \rho)^{-1 / 2}\right]^{\prime \prime} /(a \rho)^{-1 / 2}\right\} w=0 .
$$

Comparison theorems for two free-end problems, or for the free-end problem and fixedend problem associated with the same cross-section, may be stated as above, but the pointwise conditions now include the variable density $\rho$.

As our first specific example, let us consider a fixed-ended bar with a cross-section slightly perturbed from one of constant area; that is, the cross-section function is given by $a(\xi)=1+\epsilon \alpha(\xi)$, where $\epsilon>0$ and $|\epsilon| \ll 1$. Carrying out the approximation through first-order terms in $\epsilon$, we find $\left(a^{1 / 2}\right)^{\prime \prime} /\left(a^{1 / 2}\right) \sim(1 / 2) \epsilon \alpha^{\prime \prime}(\xi)$. Thus if the graph of the cross-section is concave up, $\alpha^{\prime \prime}>0$, the frequencies will be greater than or equal to those of the uniform bar. On the other hand, if the graph is concave down, the inequality will be reversed. Similarly, for the free-ended, slightly perturbed, uniform bar, we have $\left(a^{-1 / 2}\right)^{\prime \prime} /\left(a^{-1 / 2}\right) \sim(-1 / 2) \epsilon \alpha^{\prime \prime}(\xi)$. Thus, if the graph of the cross-section is concave up, the frequencies will be less than or equal to those of the uniform bar. If the graph is concave down, the inequality will be reversed.

As a second example, consider the eigenvalue problem prescribed by Eqs. (4) and (5), or Eqs. (9) and $\left(9^{\prime}\right)$, and set

$$
a(\xi)=\exp (c \xi) .
$$

Eqs. (4) and (9) both become $w^{\prime \prime}+\left(\lambda-c^{2} / 4\right) w=0$ and the boundary conditions (5) and $\left(9^{\prime}\right)$ are unchanged. Thus the eigenvalues for both the fixed-end and free-end problems, corresponding to the cross-section given by Eq. (10), are the same as those for a uniform bar, each increased by $c^{2} / 4$. Moreover, since $(\log a)^{\prime \prime}=0$, the eigenvalues for the fixed-end and free-end problems are identical. 
Our third example is a fixed-ended bar in the form of a solid of revolution. The cross-section function is given by $a(\xi)=K r^{2}(\xi)$, where $K$ is a constant. If we were to consider a uniform expansion or a uniform contraction, that is, if we were to replace $r$ by $r+c$, where $c$ is a constant, $\left(a^{1 / 2}\right)^{\prime \prime} /\left(a^{1 / 2}\right)=r^{\prime \prime} /(r+c)$. Thus if $c r^{\prime \prime}$ is positive, the frequencies will be less than or equal to those of the original bar; and, conversely, if $c r^{\prime \prime}$ is negative, the eigenvalues will not be decreased. A parallel calculation for the freeend case shows that exactly the same conclusions hold true.

As our final example, we again consider the fixed-end case and note that if $a(\xi)=$ $(M \xi+N)^{2}$, where $M$ and $N$ are constant, then $\left(a^{1 / 2}\right)^{\prime \prime}=0$. Eqs. (4) and (5) then imply that the frequencies for a truncated circular cone are exactly the same as those for a uniform bar. This result may seem somewhat more natural if one observes that the solution to Eq. (1) for the above choice of $a(\xi)$ can be found explicitly [3] to contain half-order Bessel functions which, in turn, lead to the same trigonometric transcendental equation for the eigenvalues of the uniform bar. We also observe that if we set

$$
a(\xi)=(M \xi+N)^{-2},
$$

in the free-end case, the frequencies will be the same as those of a uniform bar with free ends.

This example leads to a slightly more general question. Given a shape function $b(\xi)$ such that $b^{1 / 2}>0$ for $0 \leqq \xi \leqq 1$, can we find a linearly independent shape function $a(\xi)$ such that $a^{1 / 2}>0$ on $0 \leqq \xi \leqq 1$ and such that the eigenvalues of the two consequent problems, Eqs. (4) and (5), are the same? We see from Eqs. (4) and (7), that this will be the case if

$$
\left(a^{1 / 2}\right)^{\prime \prime}-\left[\left(b^{1 / 2}\right)^{\prime \prime} /\left(b^{1 / 2}\right)\right]\left(a^{1 / 2}\right)=0 .
$$

If the question is to be answered affirmatively, we must find two linearly independent solutions of the second-order differential equation (11). Obviously, one such solution is $a^{1 / 2}=b^{1 / 2}$. A second, linearly independent solution can be found, by the method given in [4], to be

$$
[a(\xi)]^{1 / 2}=[b(\xi)]^{1 / 2} \int_{0}^{\xi}[1 / b(t)] d t+[b(\xi)]^{1 / 2} .
$$

We therefore see that, given all of the frequencies of longitudinal oscillation of a fixedended bar, it is impossible to determine the shape uniquely since there are at least two shapes having the same set of frequencies. It is also clear that one can find two independent cross-sections which will yield the same frequencies for the free-end case simply by using the previous argument with $a$ replaced by $a^{-1}$ and $b$ by $b^{-1}$.

Acknowledgment. This work has been partially supported by the National Science Foundation under Grant Number GP-9453.

\section{REFERENCES}

[1] Tien-Yu Tsui, Wave propagation in a finite length bar with variable cross-section, J. Appl. Mech. 35, 824-825 (1968)

[2] R. Courant and D. Hilbert, Methods of mathematical physics. Vol. 1, Interscience, New York, 1953

[3] F. B. Hildebrand, Advanced calculus for engineers, Prentice-Hall, Englewood Cliffs, N. J. 1955, pp. $165 \mathrm{ff}$.

[4] W. E. Boyce and R. C. DiPrima, Elementary differential equations and boundary value problems, 2d ed., Wiley, New York, 1969, pp. 103-104 